Fetal Diagnosis and Therapy

\title{
Making Decisions When No Good Choices Exist: Delivery of the Survivor after Intrauterine Death of the Co-Twin in Monochorionic Twin Pregnancies
}

\author{
Mark I. Evans $^{\text {a T.K. Lau }}{ }^{\mathrm{b}}$ \\ ${ }^{a}$ Fetal Medicine Foundation of America, Comprehensive Genetics, and Mt. Sinai School of Medicine, New York, N.Y., \\ USA; ${ }^{b}$ Maternal Fetal Medicine Unit, Department of Obstetrics and Gynecology, The Chinese University of \\ Hong Kong, Hong Kong, SAR, China
}

\section{Key Words}

Twins $\cdot$ Fetal demise $\cdot$ Risk to survivor $\cdot$ Neurologic

impairment $\cdot$ Technology assessment

\begin{abstract}
Introduction: Infertility therapies have markedly increased the incidence of multiple pregnancies and concomitantly monozygotic twinning. In utero death of one monozygotic twin predisposes the survivor to death and serious morbidity from hemodynamic collapse, thromboplastins, or microemboli. The optimal management of such cases, however, is unclear. Methods: We compared single-twin demise to similar clinical conundrums in the past to try to derive a model for the data needed to reach a consensus. Results: The management of such pregnancies is very controversial as the clinician must balance the risks of damage to the survivor by temporizing against the risks of prematurity and the possible futility of damage already having occurred because it is impossible to precisely time and document when the damage occurs. While there is not yet a standard consensus, the trend is towards more aggressive management either by rapid delivery or transfusion to ameliorate possible damage, but the risks are still very high for the survivor. Conclusions: The trend is moving towards more aggressive management
\end{abstract}

\section{KARGER}

Fax +4161306 1234

E-Mail karger@karger.ch

www.karger.com (c) 2010 S. Karger AG, Basel

$1015-3837 / 10 / 0284-0191 \$ 26.00 / 0$

Accessible online at:

www.karger.com/fdt than previously. We pose several questions that still remain to be answered to derive a consensus as to management, but it is unlikely that the required data could ever be obtained in a rigorous, randomized fashion.

Copyright $\odot 2010$ S. Karger AG, Basel

\section{Introduction}

There are many situations in medicine in which there is a clinical fork in the road, and a path has to be taken. In many of these, there are considerable, deleterious consequences to any choice taken. In reproductive medicine, there is a litany of examples. Classic conundrums have included choice of route of delivery, e.g. cesarean section versus forceps or vacuum, management of breeches, premature rupture of membranes, how many embryos to transfer in in vitro fertilization and the resultant need to consider multifetal pregnancy reduction, and balancing aggressiveness in cancer therapy versus its side effects and the patient's quality of life.

This review also contains a lot of the authors' personal opinions.
Mark I. Evans, MD

Comprehensive Genetics

131 East 65th Street

New York, NY 10065 (USA)

Tel. +1 212288 1422, Fax +1 212879 2606, E-Mail Evans@ Compregen.com 
With the explosion of infertility therapies has come a profound increase in the occurrence of multiple gestations - a significantly increased proportion of which are monozygotic [1]. The literature has chronicled the increasing risks of monozygotic gestations, including considerably higher risks of pregnancy loss, congenital abnormalities - particularly defects of laterality, twin-totwin transfusion, selective intrauterine growth restriction, and high risk of death and damage to the survivor [1]. As far back as the 1960s and updated for decades, hypotheses to explain the pathogenesis of damage were proposed and have evolved. Benirschke and Kaufmann's [2] hypothesis of thromboemboli and thromboplastins from the demised fetus to the survivor has been supplanted in part by a theory of a 'vascular sink' being created by the lack of pressure in the demised placenta, allowing a rush of blood flow into it from the survivor, which then produces hypovolemic shock and hypoxia [3].

Regardless of the mechanism, there has never been a dispute as to the existence of high risks. Most recent estimates suggest that for a mid- or third-trimester spontaneous demise, the risk of death to the other twin is about $12 \%$, and the risk of neurological damage may be closer to $20 \%$ [4]. Since it is usually unclear exactly when damage or death may occur, the obstetrical management of such cases has been extremely complex and fraught with danger for mother and fetus and medico-legal risks when outcomes are poor. Some have argued that irreparable damage may occur very soon after the demise of the cotwin, and others have believed that there is a period of time before such a damage is likely [1]. There is no way to conclusively prove to a near $100 \%$ certainty the timing of damage, and it is unlikely that either animal models or epidemiologic data will do so in the near future.

How fast to deliver then becomes a conundrum with no certain pathway for the clinician and patient to follow.

\section{Relevant Predicates}

Trying to evolve a consensus on such a difficult topic first requires assessment of the risks for the situation being debated and asking the question: 'have we been down this road before, and are previous comparable situations instructive?' In general, the more likely the adverse outcome, the greater the risk tolerated to attempt to avoid it. Amniocentesis and chorionic villus sampling - even when their risks were believed to be close to $2 \%$ - were considered reasonable approaches to find recessive genetic disorders with a $25 \%$ incidence. As the procedure risks fell below 1\%, a chromosomal abnormality risk of $0.5 \%$ (35 year-olds) to warrant them became commonplace [5].

Fetal surgery was developed in the 1980s for congenital diaphragmatic hernia. It had many failures before even the first success. The National Institute of Health trial, which predicted a $70 \%$ surgical versus a $35 \%$ control success rate, was stopped prematurely, and the surgical procedure was abandoned when the control (neonatal management only) outcomes appeared to be much closer to the surgical results than was anticipated [6]. For meningomyelocele, the primary issue for prenatal intervention was quality of life versus life or death as previously required for open fetal procedures. Preliminary longterm data show that there may be enough improvement in neuromotor function to warrant intervention resulting in an ongoing prospective randomized trial comparing prenatal to postnatal neurosurgical closure for this anomaly [7].

Mid-forceps and breech deliveries have largely disappeared from the obstetric armamentarium as a $1-4 \%$ association with fetal/neonatal damage/death are generally considered now to be too high in comparison to the risks of cesarean section. Similarly, vaginal births after a cesarean section have largely come and gone over concerns of uterine rupture and its consequences especially for the fetus.

Multifetal reduction was begun in the 1980s for gestations in which the likelihood of survival was considered low to nonexistent [1]. As experience on thousands of cases has emerged, the indications have loosened considerably to where the vast majority of cases involve minimizing the morbidities as much as the mortalities.

Over the years, we have extensively studied how patients and their doctors try to make decisions in situations of great concern and controversy [8]. Patients can be divided into those that have a 'medical' frame, i.e. they value hard data most in trying to reach a conclusion; those who have a 'conceptional' frame, who value religious beliefs primarily, and those who have a 'lifestyle' frame, whose primary motivation is to reduce morbidity the most - even at the expense of increased mortality. Of course, at least the first and third models require an adequate estimation of the underlying risks to help direct the decision-making process.

What all of the above situations had in common was a period of considerable uncertainty as to management that was only 'solved' by the acquisition of data. Only after a relevant database had been acquired could a generalized consensus in the literature and eventually profes- 
sional societies emerge. There is an entire literature on the process of technology assessment, which fills articles, books, society meetings, government agencies, and insurance company and court files [9].

In general, there is a period of 'development' in which a small number of investigators - commonly but not always at universities, working under institutional review board imprimatur - develop and begin to publish a new approach [9]. For fetal reduction and other approaches, the methodology of a new approach to any significant problem eventually works its way into tertiary or even only 'quaternary' practice. Over time with experience, comfort develops, statistics emerge, and demand for the new approach increases. Availability begins to expand, and new physicians begin to try the procedure. This expansion of availability is referred to as the 'period of diffusion', at which time utilization skyrockets, but so do complications. Eventually, equilibrium is reached in which there is reasonable understanding of risks and benefits, and the procedure is available to large numbers of patients. It is often only at the end of this course that insurance coverage allows for true widespread access for patients [9].

How does all the above apply to the surviving twin? The unifying theme from the cited examples is that there is always a period of uncertainty as to how to improve an unsatisfactory status quo that has multiple ups and downs in trying new approaches. This is exactly where we are with the management of the surviving twin.

\section{The Surviving Twin}

Evidence so far suggests that damage to the surviving twin mainly derives from consequences of hemodynamic disturbance secondary to acute hemorrhage into the demised fetus through vascular communications [3]. Such hemodynamic disturbances are similarly believed to be at least partly responsible for the residual risks following laser vessel ablation treatments for twin-to-twin transfusions - possibly because of incomplete occlusion - with recent data suggesting a 6-9\% risk of neurologic impairments $[4,10,11]$. Even if we assume the 'simple' mechanism of hypovolemia and its consequences, including hypoxia and hypoperfusion, to be the main mechanism affecting the welfare of the surviving twin, there are still many unanswered questions making a logical decision on management impossible.

There are many factors which affect the rate of acute fetal-fetal hemorrhage after the death of one of the twins.
These include the number, the size, and the type of vascular anastomoses. In most cases, we do not know the answer to these questions, and therefore we are unable to predict the consequences with any precision.

If there are major placental vascular communications, the death of one twin will probably lead to immediate and severe hypotension, hypovolemia and hypoxia in the other, resulting in its immediate death. It is unlikely that any intervention would save the co-twin. With fewer and smaller vascular anastomoses, the rate of exsanguination would likely be slower, but we do not know the rapidity of deterioration or the timing of maximum effect. Although we can now predict the degree of fetal anemia with reasonable accuracy with Doppler ultrasound, we do not have a reliable way of assessing the degree of fetal hypotension or hypoxia.

With time, there will be thromboses of the vascular anastomoses, and eventually fetal-fetal hemorrhage will stop. If the co-twin could survive until then, subsequent death is probably less likely, and it will gradually recover although irreversible neurological damage may have occurred at the time of maximum hemodynamic disturbance. However, we do not know how quickly these vascular channels stop functioning after a co-twin's death. In those cases that had previously undergone laser therapy with functional separation of the placentas for severe twin-to-twin transfusion syndrome, the vascular 'sink', thromboembolus, or thromboplastin mechanisms would seem not to apply except if the occlusion were incomplete. With current methods, it would seem impossible to discern the exact etiology or the timing of neurologic impairment in such cases.

Recently, there has been an increasing interest in monitoring the surviving twin by Doppler ultrasound and to transfuse those with evidence of fetal anemia [11]. We must realize that this approach only addresses the secondary effect of hemorrhage. Assuming analogy to the adult and child, hemorrhage and hypotension will not cause immediate reduction in hemoglobin level, but the subsequent expansion of plasma volume and hemodilution will. This management approach will not be useful in preventing early complications, and may even not be necessary in those fetuses in which hemorrhage has already stopped and stabilized. However, we do not have effective clinical tools to tell us where in the timeline of the pathophysiologic process the surviving twin is situated at any particular moment.

Fetal maturity obviously is another important determining factor. It is well accepted that premature fetuses are more susceptible to insults leading to neurological 
and other long-time complications. However, again, we do not have reliable methods to predict or determine the degree of fetal damage in utero.

In short, it is likely that in some cases damage to the co-twin can occur within minutes to the first few hours after the death of one twin. The window for potentially effective treatment is, thus, very narrow. Given that the precise timing of death of a co-twin is usually unknown, any attempt in deriving an evidence-based management approach would be close to impossible.

Because of these many difficulties and limitations stated above, we can only adopt a general approach of risk reduction in managing a monochorionic twin pregnancy with a single fetal death. Risk reduction for the co-twin at a gestational age ordinarily with reasonable expectation of intact survival can be either by delivery or by 'rescue' transfusion as soon as possible after the first twin's presumed death. Survival at all gestational ages has improved markedly, and by 32 weeks, the intact survival in most advanced nurseries of a fetus in good health is arguably so high as to make delivery a preferred option even when the fetus is in no apparent distress [12]. With evidence of possible threats to fetal health, the balance would seem to tilt towards expeditious delivery. If damage seems to be confirmed, e.g. acute substantial hemorrhage, then the situation becomes even more complex, with no obvious satisfactory medical answer. Likewise, at earlier gestational ages, when the compound effects of prematurity superimpose upon the potential damage to the co-twin, then focusing on attempted amelioration by transfusion seems more reasonable.

\section{Conclusions}

There are considerable gaps in our knowledge base; coalescing on any single strategy from an 'evidence-based medicine' approach is thus impractical at best and impossible in reality. Furthermore, some problems cannot be assessed by the gold standards of randomized trials or animal experiments.

The types of questions that need to be asked, but probably cannot be answered, include:

(1) What types of damage occur and what is the timing of potential damage to the survivor before the demise of the co-twin, stratified by categorization of the clinical situation that led to the demise?

(2) Are there markers or exams such as MRI or cordocentesis that could be reliably used to assess risk to the survivor before or after demise of the co-twin?
(3) Do these markers and their interpretation differ from those in use for singletons?

(4) Is there any way to determine the extent of any 'window of opportunity' to intervene to improve outcomes?

Under these circumstances what is needed is a real, multi-institutional database of contemporaneously managed cases with modern methods of evaluation such as MRI and other sophisticated imaging techniques, standardization of data, and proper follow-up of outcomes. Unfortunately, because under virtually all circumstances there is no precise way of determining the timing of demise or compromise before death, it will be virtually impossible to develop truly rigorous models. Likewise, animal models will be of limited use in predicting less than severe neurologic impairments.

In the predicate situations described earlier, there was in some cases (e.g. fetal surgery) the ability to establish animal models. In others (e.g. breech deliveries) there was the ability to prospectively randomize patients in essentially identical situations to different treatment arms. Others, such as multiple pregnancies and fetal reduction, had to rely upon multicenter collaborations of epidemiologic data, but there could be significant matching of numerous obvious variables around which to build comparisons.

Unfortunately, just saying we should get better data does not help the clinician faced with the problem today. Our assessment of the data suggests that, when a fetus is beyond 32 weeks, the co-twin's death seems recent, and there is evidence or reasonable suggestion of impending but not yet irreparable damage such as fetal monitor changes, expeditious delivery would seem warranted. As one moves earlier in pregnancy, demise becomes remote from observation, and fetal status is either apparently completely normal or very abnormal, rushing to delivery is less likely to be the best management. Temporizing methods might be the best under such circumstances.

The data do not yet warrant blanket statements about a single management approach. However, we believe the curve is shifting towards a more aggressive philosophy to intervene earlier and faster when the demise of a monozygotic twin is documented. Regardless of whatever decisions are reached by the medical team or patient, a thorough discussion with the couple, and in the chart, is critical to avoid second guessing in scenarios in which the likelihood of a poor outcome is high regardless of the path chosen. 


\section{References}

1 Evans MI, Ciorica D, Britt DW, Fletcher JC: Reduction in multiple pregnancies; in Evans MI, Johnson MP, Yaron Y, Drugan A (eds): Prenatal Diagnosis. New York, McGraw Hill, 2006, pp 561-570.

2 Benirschke K, Kaufmann P: Pathology of the Human Placenta, ed 2. New York, Springer, 1990.

3 van Gemert MJC, Nikkels RGJ: Placental vasculature in the pathogenesis and morbidity in multiple pregnancies; in Blickstein I, Keith LG (eds): Multiple Pregnancy. London, Taylor \& Francis, 2005, pp 586-588.

4 Ortibus E, Lopriore E, Deprest J, Vandenbussche FP, Walther FJ, Diemert A, et al: The pregnancy and long term neurodevelopmental outcome of monochorionic diamniotic twin gestations: a multicenter prospective cohort study from the first trimester onward. Am J Obstet Gynecol 2009;200:494.e1-494. e8.
5 Drugan A, Evans MI: Amniocentesis; in Evans MI, Johnson MP, Yaron Y, Drugan A (eds): Prenatal Diagnosis. New York, McGraw, 2006, pp 415-422.

6 Harrison MR, Keller RL, Hawgood SB, Kitterman JA, Sandberg PL, Farmer DL, et al: A randomized trial of fetal endoscopic tracheal occlusion for severe fetal congenital diaphragmatic hernia. N Engl J Med 2003;349: 1916-1924.

7 Danzer E, Gerdes M, Bebbington MW, Sutton LN, Melchionni J, Adzick NS, Wilson RD, Johnson MP: Lower extremity neuromotor function and ambulation potential following in utero myelomeningocele repair. Fetal Diag Ther 2009;25:47-53.

-8 Britt DW, Evans WJ, Mehta SS, et al: Framing the decision: determinants of how women considering MFPR as a pregnancy management strategy frame their moral dilemma. Fetal Diag Ther 2004;19:232-240.
9 Cohen AB, Hanft RS: Technology in American Health Care: Policy Directions for Effective Evaluation and Management. Ann Arbor, University of Michigan Press, 2004.

10 Rossi AC, D’Addario V: Laser therapy and serial amnioreduction as treatment for twintwin transfusion syndrome. Am J Obstet Gynecol 2008; 198:147-152.

11 Quarello E, Stirnemann J, Nassar M, Nasr B, Bernard JP, Leleu-Huard F, Ville Y: Outcome of anaemic monochorionic single survivors following early intrauterine rescue transfusion in cases of feto-fetal transfusion syndrome. Br J Obstet Gynaecol 2008;115:595601.

12 Fanaroff AA, Stoll BJ, Wright LL, Carlo WA, Ehrenkranz RA, Stark AR, et al: Trends in neonatal morbidity and mortality for very low birthweight infants. Am J Obstet Gynecol 2007;196:147.e1-147.e8. 